\title{
Optical Coherence Tomography Angiography Evaluation of Inherited Retinal Dystrophies - a Literature Review
}

\author{
Francesco Romano, Alessandro Arrigo and Maurizio Battaglia Parodi \\ Department of Ophthalmology, Vita-Salute University, Scientific Institute San Raffaele, Milan, Italy
}

DOl: https://doi.org/10.17925/EOR.2018.12.1.45

$\mathrm{T}$

he primary outcome of this article was to review the main vascular alterations occurring in inherited retinal dystrophies described in literature. Secondary outcomes included the discussion of the potential clinical and pathophysiological implications of such vascular impairment.

\section{Keywords}

Optical coherence tomography angiography (OCT-A), inherited retinal dystrophies, retinitis pigmentosa, Stargardt disease, best vitelliform macular dystrophy, X-linked retinoschisis

Disclosure: Francesco Romano, Alessandro Arrigo and Maurizio Battaglia Parodi have nothing to declare in relation to this article.

Review Process: Double-blind peer review.

Compliance with Ethics: This study involves a review of the literature and did not involve any studies with human or animal subjects performed by any of the authors.

Authorship: All named authors meet the International Committee of Medical Journal Editors (ICMJE) criteria for authorship of this manuscript, take responsibility for the integrity of the work as a whole, and have given final approval to the version to be published. open Access: This article is published under the Creative Commons Attribution Noncommercial License, which permits any non-commercial use, distribution, adaptation and reproduction provided the original author(s) and source are given appropriate credit.

(C) The Authors 2018

Received: 12 January 2018

Accepted: 26 February 2018

Citation: European Ophthalmic Review. 2018;12(1):45-9

Corresponding Author: Francesco Romano,

Department of Ophthalmology, Scientific Institute San

Raffaele, Vita-Salute University, Via Olgettina,

60, 20132, Milan, Italy.

E:fromano@studenti.unisr.it

Linkedın: https://it.linkedin.com/in/

francesco-romano-9a3a53141

Support: No funding was received in the publication of this article.
Inherited retinal dystrophies (IRD) are genetic disorders characterised by variable, albeit progressive degeneration of the retina, retinal pigment epithelium (RPE) and choriocapillaris (CC). ${ }^{1.2}$ Prevalence varies in different countries, affecting approximately 1 in 4,000 individuals, with symptoms including poor peripheral or night vision, metamorphopsias, reduced central vision or blindness. ${ }^{1.3}$ Their pattern of inheritance may be autosomal recessive (e.g. Stargardt disease), autosomal dominant (e.g. Best disease), X-linked (juvenile retinoschisis and choroideremia) or mixed (e.g. retinitis pigmentosa, with all its phenotypes). Chorioretinal dystrophies also present a high degree of clinical, pathophysiological and histological findings, making their diagnosis and management occasionally challenging. ${ }^{4-9}$ In this regard, the use of multimodal imaging and genetic characterisation are increasingly recognised as fundamental instruments in the assessment of patients affected by these disorders. ${ }^{10-13}$

Optical coherence tomography (OCT) angiography (OCT-A) represents a functional extension of OCT technology, employing motion contrast from blood flow rather than simple reflectance intensity to acquire, in a few seconds, high-resolution information about ocular vessels. ${ }^{14} \mathrm{Although}$ fluorescein angiography and indocyanine green angiography are still considered the gold standards for the assessment of all the retinal vascular diseases, OCT-A is progressively gaining rapid and positive consensus among ophthalmologists as a valid alternative or integrative technique to the previously mentioned angiographic modalities. ${ }^{15,16}$

For these reasons, OCT-A is one of the most recent and utilised tools to investigate the presence and the extent of the vascular alterations in IRD. ${ }^{17-18}$ Its advantages include the possibility to analyse the vascular profile of these patients in a non-invasive (dye-less) and rapid (few seconds) manner, devoid of adverse effects. Moreover, OCT-A allows a depth-resolved study of retinal vasculature in patients who would not be the best candidates for other angiographic examinations, including those with renal disorders, pregnant women and those with hypersensitivity reactions. Even though vascular impairment has been described in several dystrophies, no specific review has thoroughly analysed the different vascular involvement in the main chorioretinal dystrophies or focused on their clinical implications and future perspectives.

In recent years, numerous OCT-A studies have focused on the extent and evolution of vascular impairment in IRD, evaluating their relationships with the fundus findings, best-corrected visual acuity (BCVA) outcome and spectral domain optical coherence tomography (SD-OCT). The most common parameters included vessel density (expressed as percentages, \%) and foveal avascular zone area (FAZ, expressed as $\left.\mathrm{mm}^{2}\right)^{19-33}$

The primary outcome of this article is to review the main vascular alterations occurring in IRD described in literature. Secondary outcomes include the discussion of the potential clinical and pathophysiological implications of such vascular impairment.

\section{Retinitis pigmentosa}

The diagnosis of retinitis pigmentosa (RP) includes a clinically and genetically heterogeneous group of IRD, principally characterised by the progressive dysfunction of rod photoreceptors 
Figure 1: Optical coherence tomography angiography features of retinitis pigmentosa

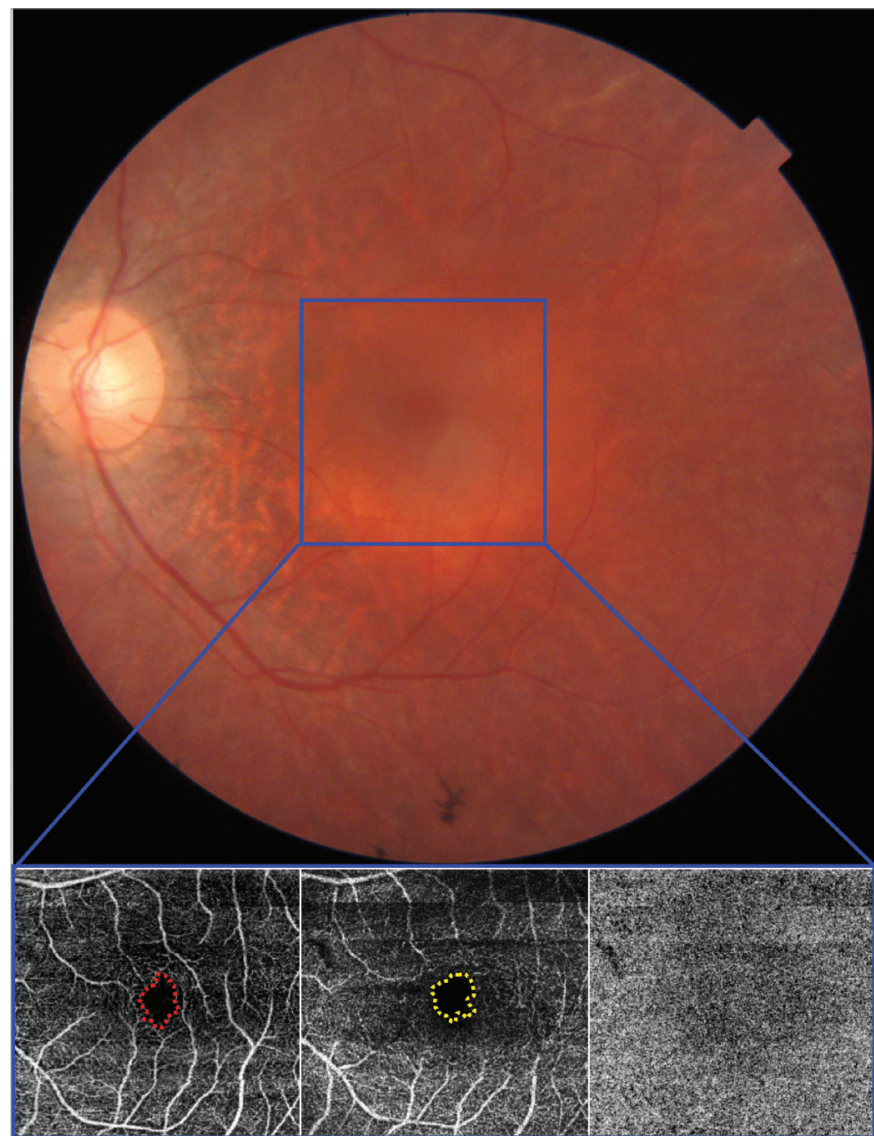

Optical coherence tomography angiography using swept source Triton DRI OCT (Topcon Corporation, Tokyo, Japan). Top: Colour fundus photograph shows the typical fundus aspect characterised by diffuse chorioretinal atrophy and bone-spicule pigmentation in the inferior mid-periphery. Bottom: Optical coherence tomography angiograms sequentially show, from left to right, the vascular alterations occurring at the SCP, DCP and CC. Vascular rarefaction is especially visible in the foveal region of the SCP and DCP, while CC seems unaffected. Foveal avascular zone areas of DCP appears slightly enlarged (yellow dashed lining).

$C C=$ choriocapillaris; $D C P=$ deep capillary plexus: $S C P=$ superficial capillary plexus

and followed by secondary degeneration of cones and retinal pigment epithelium. ${ }^{34}$

Of note, the appearance of the superficial capillary plexus (SCP) and deep capillary plexus (DCP) seems qualitatively altered, this finding being especially marked in the temporal sector of these patients. ${ }^{19}$ These results have indeed been confirmed by some quantitative studies, disclosing a general rarefaction of the retinal plexuses when compared with control subjects, both in the foveal and para-foveal areas. ${ }^{19-21}$ Interestingly, no study has identified significant damages in the CC plexus of these patients. With regard to the FAZ area, a meaningful enlargement was exclusively described at the level of the DCP, especially in the presence of visual acuity deterioration. ${ }^{19,20}$ Figure 1 shows the typical vascular changes of patients with RP.

\section{Stargardt disease}

Stargardt disease (STGD) is considered to be the most frequent recessive macular dystrophy, resulting from the accumulation of lipofuscin in the RPE and leading to secondary photoreceptor dysfunction. ${ }^{35,36}$ Patients are generally homozygous for $A B C A 4$ gene mutations.

From a qualitative point of view, signs of vascular rarefaction are particularly evident in the temporal area, both in the SCP and the DCP;
Figure 2: Optical coherence tomography angiography features of Stargardt disease

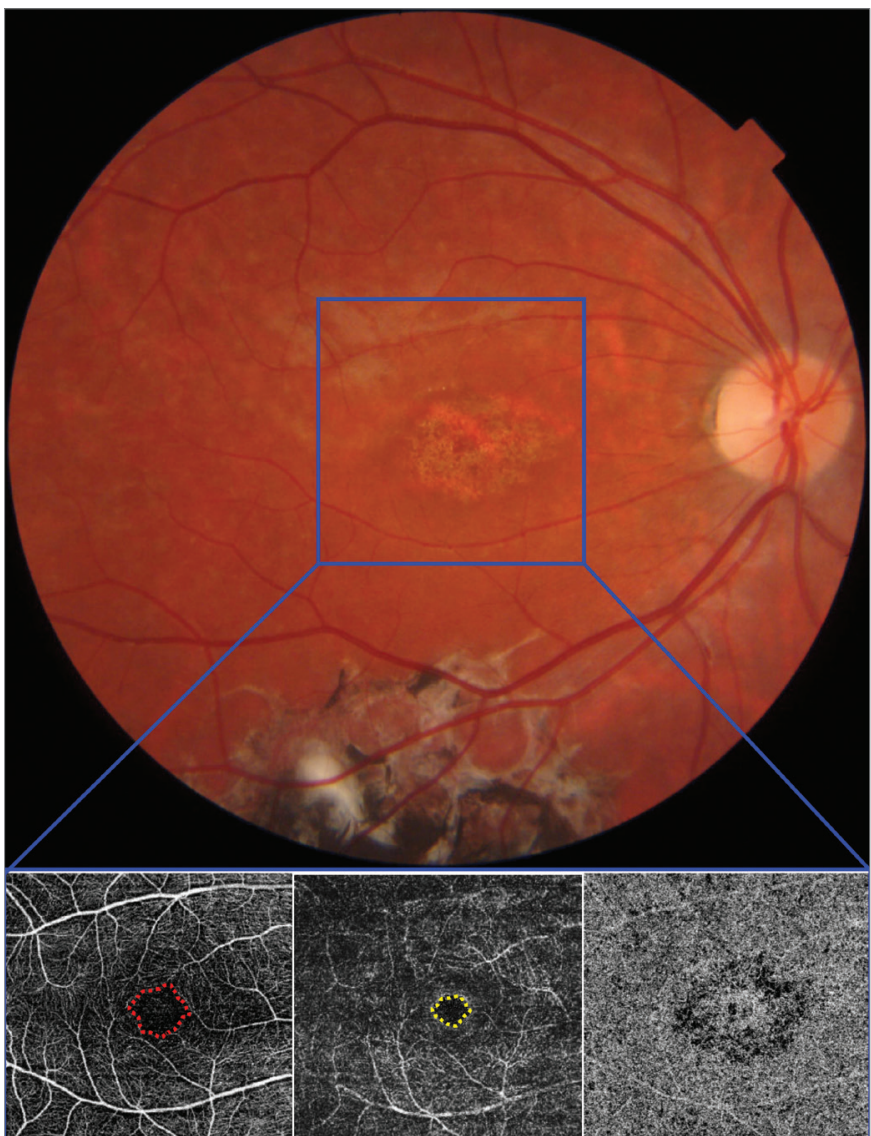

Optical coherence tomography angiography using swept source Triton DRI OCT (Topcon Corporation, Tokyo, Japan). Top: Colour fundus photograph shows the typical fundus aspect with yellow flecks and fine granularity at the posterior pole. Along the inferior vascular arcade, the signs of subretinal fibrosis and retinal pigment epithelium hyperplasia in STGD after a mild blunt trauma are visible. Bottom: The OCT angiograms present, from left to right, the vascular alterations occurring at the SCP, DCP and CC. Superficial capillary plexus and DCP disclose a generalised rarefaction, whereas CC appears rarefied in correspondence with the granular deposits, probably due to masking effect. Foveal avascular zone areas of SCP and DCP are highlighted in red and yellow respectively.

$C C=$ choriocapillaris; $D C P=$ deep capillary plexus; $O C T=$ optical coherence tomography; SCP = superficial capillary plexUS; STGD = Stargardt disease.

moreover, the lipofuscin-filled flecks seem to exert a clear masking effect on the underlying $\mathrm{CC}$ layer. ${ }^{22} \mathrm{~A}$ few quantitative analyses have supported this reduced vessel density in both SCP and DCP when compared to controls, whereas vessel density does not appear to be affected in the CC of patients with STGD.22-4 Nevertheless, in the subgroup of patients with SD-OCT-documented macular atrophy, a marked rarefaction is also detected in the $\mathrm{CC}^{22,23} \mathrm{An}$ enlargement of the FAZ area is instead appreciable at the level of SCP. ${ }^{22}$ Figure 2 illustrates the vascular damage occurring in eyes with Stargardt disease.

\section{Adult-onset foveomacular vitelliform dystrophy}

Adult-onset foveomacular vitelliform dystrophy (AOFVD) is a form of pattern dystrophy characterised by bilateral, yellowish sub-foveal deposits (one-third to one disc in diameter) and by a slow clinical progression. ${ }^{37}$ Although associated with an autosomal dominant mode of inheritance (e.g. peripherin and RDS genes), a distinct genetic mutation is not detected in most cases. ${ }^{38}$

On OCT-A, a well-defined vascular rarefaction is especially identifiable at the SCP and $C^{25-7}$ Although the results from the DCP vascularity appear controversial, ${ }^{25,27}$ FAZ area seems instead to be significantly enlarged in 
Figure 3: Optical coherence tomography angiography characteristics of adult-onset foveomacular vitelliform dystrophy

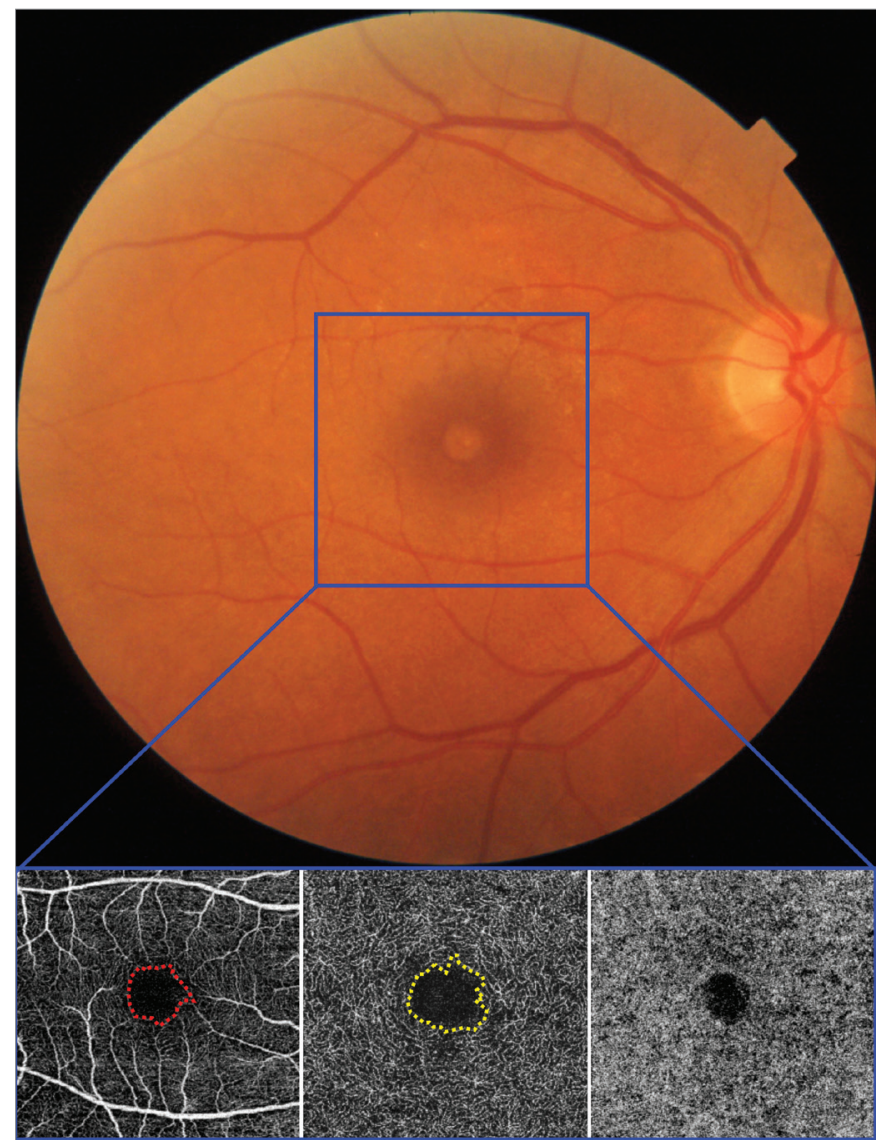

Optical coherence tomography angiography using swept source Triton DRI OCT (Topcon Corporation, Tokyo, Japan). Top: Colour fundus photograph shows the fundus appearance of a patient in the vitelliform stage of AOFVD. Bottom: Optical coherence tomography angiography scans sequentially show, from left to right, the vascular alterations OCCurring at the SCP.DCP and CC A reduced vessel density can be

identified in the SCP and the CC, especially underneath the vitelliform lesion. Foveal avascular zone area is especially enlarged in the DCP (yellow dashed line). AOFVD = adult-onset foveomacular vitelliform dystrophy; $C C=$ choriocapillaris; $D C P=$ deep capillary plexus; OCT = optical coherence tomography;

$S C P=$ superficial capillary plexus

this vascular plexus. Figure 3 shows the vascular alterations occurring in AOFVD.

\section{Best vitelliform macular dystrophy}

Best vitelliform macular dystrophy (BVMD) is a dominantly-inherited macular dystrophy, characterised by a typical egg yolk-like lesion in the early stages of the disease..$^{39}$ BEST1 is the most commonly mutated gene in this dystrophy, which, however, shows high genetic and phenotypic variability. ${ }^{6}$

A marked rarefaction can be qualitatively perceived in all the vascular layers, particularly in patients with an advanced disease; in addition, the features of choroidal neovascularisation have been reported by several groups, reaching an outstanding 36\% prevalence in the largest study currently available. ${ }^{28,29}$ Despite a clear masking effect exerted by the vitelliform material on the underlying $\mathrm{CC}$, reduced vascular density can be identified in all the vascular plexuses of patients with BVMD, with an enlargement of the FAZ area occurring at the DCP. ${ }^{29}$ In addition, the stratification of patients according to Gass' classic five stages revealed that a significant rarefaction particularly accompanies the stages following the vitelliform material disruption (stages three, four and five). ${ }^{29}$ Figure 4 shows the typical vascular changes of Best disease.
Figure 4: Optical coherence tomography angiography characteristics of Best vitelliform macular dystrophy

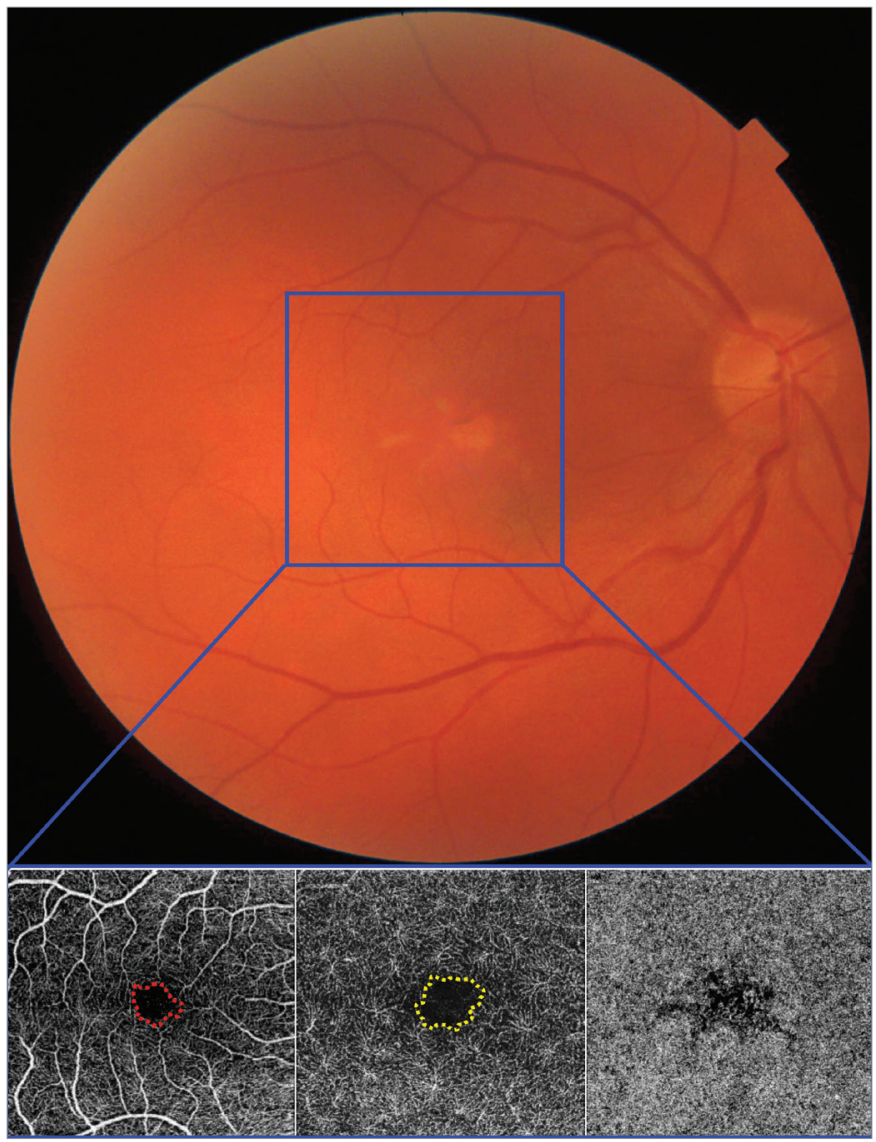

Optical coherence tomography angiography using swept source Triton DRI OCT (Topcon Corporation, Tokyo, Japan). Top: Colour fundus photograph shows the fundus appearance of a patient in the typical vitelliruptive stage of Best disease. Bottom: optical coherence tomography angiography scans sequentially show, from left to right, the vascular alterations occurring at the SCP, DCP and CC. Vascularity appears thinned in all the plexuses, whereas the foveal avascular zone area is enlarged in the DCP (yellow dashed line)

$C C=$ choriocapillaris; $D C P=$ deep capillary plexus; $O C T=$ optical coherence tomography; SCP = superficial capillary plexus

\section{Choroideremia}

Choroideremia $(\mathrm{CHM})$ is a progressive form of retinal degeneration affecting the RPE, the choroid and the outer retina. Patients typically complain about nyctalopia and decreased peripheral vision from a young age, reaching legal blindness around the third to fourth decade.40

Although little information is currently available in the literature, ${ }^{30,31}$ our experience suggests that DCP results are dramatically altered in patients with $\mathrm{CHM}$, whereas the SCP appears similar to controls. Moreover, the CC shows some vascular rarefaction exclusively limited to the area characterised by signs of chorioretinal degeneration, with the vascular alteration correlating with the extent of photoreceptor loss. ${ }^{31,32}$ Figure 5 illustrates the alterations occurring in CHM on OCT-A assessment.

\section{$X$-linked juvenile retinoschisis}

$X$-linked juvenile retinoschisis (XLJR) is a relatively common form of retinal degeneration affecting young males and mainly manifests with splitting of the outer retinal layers in the macular region. ${ }^{41}$ Although no definitive proof is available, the effective use of dorzolamide in these patients highlights a vascular role in the pathogenesis of this disease. ${ }^{42}$ 
Figure 5: Optical coherence tomography angiography features of choroideremia

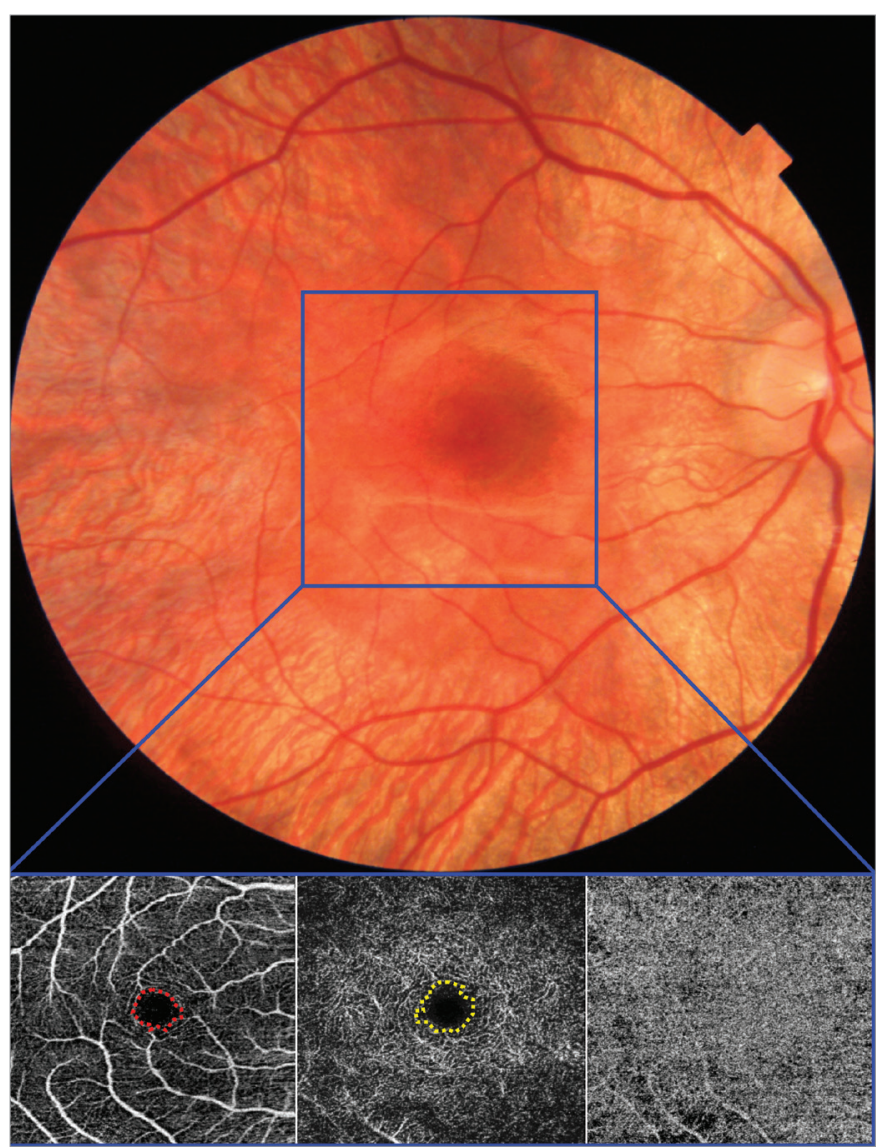

Optical coherence tomography angiography using swept source Triton DRI OCT (Topcon Corporation, Tokyo, Japan). Top: Colour fundus photograph shows the typical fundus aspect characterised by spared central retinal island, with surrounding retinal degeneration. Bottom: Optical coherence tomography angiograms sequentially show, from left to right, the vascular alterations occurring at the SCP, DCP and CC. Vascular rarefaction is clearly evident in the DCP and CC of the peripheral atrophic retina, with sparing of the unaffected macular area.

$C C=$ choriocapillaris; $D C P=$ deep capillary plexus; $O C T=$ optical coherence tomography; SCP = superficial capillary plexus.

As suggested by a recently published case series, ${ }^{33}$ our experience points toward a meaningful capillary rarefaction detectable in the DCP of patients with XLJR, with this alteration being particularly marked in the foveal area. A remarkable FAZ area enlargement can be instead observed in the same vascular plexus. Table 1 summarises the vascular alterations described in these IRDS.

\section{Discussion}

Inherited retinal dystrophies comprise a broad group of genetic disorders with variable severity and different patterns of inheritance. Although the aetiology and the evolution of these diseases are quite different, all of them result in an irreversible loss of visual acuity and, as a consequence, a poor quality of life. $3 ., 6,6,8$

Optical coherence tomography angiography is a relatively new imaging tool that allows a quick and non-invasive assessment of retinal and choroidal vascularisation. Since its introduction, OCT-A has been increasingly employed to describe the capillary alterations occurring
Table 1: Graphical representation of the vascular impairment occurring in the different inherited retinal dystrophies

\begin{tabular}{|l|l|l|l|l|l|l|}
\hline & \multicolumn{2}{c}{ Vessel density } & \multicolumn{2}{l}{ Foveal avascular zone } \\
& SCP & DCP & CC & \multicolumn{1}{l|}{ SCP } & DCP \\
\hline Retinitis pigmentosa & $\downarrow$ & $\downarrow$ & $=$ & & $=$ & $\uparrow$ \\
\hline Stargardt disease & $\downarrow$ & $\downarrow$ & $=/ \downarrow$ & $\uparrow$ & $=$ \\
\hline AOFVD & $\downarrow$ & $=$ & $\downarrow$ & & $=$ & $\uparrow$ \\
\hline Best disease & $\downarrow$ & $\downarrow$ & $\downarrow$ & & $=$ & $\uparrow$ \\
\hline Choroideremia & $=$ & $\downarrow$ & $\downarrow$ & & $=$ & $=$ \\
\hline XLIR & $=$ & $\downarrow$ & $=$ & & $=$ & $\uparrow$ \\
\hline
\end{tabular}

$\downarrow$ : vascular rarefaction; $=$ : unaffected plexus; $\uparrow$ : enlarged foveal avascular zone AOFVD = adult-onset foveomacular vitelliform dystrophy; $C C=$ choriocapillaris; $D C P=$ deep capillary plexus; $S C P=$ superficial capillary plexus; $X L J R=X$-linked juvenile retinoschisis.

in patients with various retinal disorders, opening the scene to new explanations of their pathogenesis. Moreover, in the last few years new approaches have been proposed in the field of IRD to improve their prognosis through early phenotypic characterisation, the prompt identification of treatable complications and the advancement of innovative therapies. ${ }^{43-47}$

Currently available data reveal that a marked reduction of the vessel density is noticeable from the earliest stages in most IRD; therefore the manifestation of these microvascular alterations might be implicated in the pathogenesis of these diseases as well. Even if the rarefaction observed by means of OCT-A was simply secondary to the damage occurring in the outer retina or RPE of these patients, the worsening functional outcomes or the boosting of the natural evolution of the disease are still imputable to the resulting vascular damage. Another clinically useful application of this technique regards the superior ability to identify macular choroidal neovascularisation in the presence of RPE-derived pigment exerting a clear masking effect above the underlying growing new vessels.

In addition, as in the last decade a rising attention has been progressively devoted to gene therapy approaches to treat these disorders (e.g. CHM and RP), OCT-A evaluation might add valuable information and act as a precise technique to pre-operatively assess the best sites for the injection of viral vectors or to indirectly evaluate the outcomes of this gene transfer from a vascular perspective..$^{48,49}$

In conclusion, we acknowledge that there are several limitations to the currently available studies of IRD conducted by means of OCT-A, including the limited number of studies and patients present in the literature and the high variability found even within the different phenotypes of the same disease. Additional disputable aspects are characterised by segmentation or projection artefacts induced by the morphological alterations occurring in the retinal layers, making the microvascular assessment, to some extent, inaccurate. ${ }^{50}$ Therefore, further studies including larger cohorts and longer follow up are needed, along with a more detailed functional assessment, to better characterise the vascular profile of IRD and to further promote OCT-A as a well-established imaging tool in this field. $\square$
Gass JD. Stereoscopic atlas of macular diseases: diagnosis and treatment, 4th ed. St Louis, MO: Mosby, 1997.

Bravo-Gil N, Méndez-Vidal C, Romero-Pérez L, et al. Improving the management of inherited retinal dystrophies by targeted sequencing of a population-specific gene panel Sci Rep. 2016;6:23910
Hartong DT, Berson EL, Dryja TP. Retinitis pigmentosa. Lancet. 2006;368:1795-809.

4. Rotenstreich Y, Fishman GA, Anderson RJ. Visual acuity loss and clinical observations in a large series of patients with Stargardt disease. Ophthalmology. 2003;110:1151-8.

5. Mrejen S, Audo I, Bonnel S, Sahel JA. Retinitis pigmentosa and other dystrophies. Dev Ophthalmol. 2017;58:191-201.

6. Boon CJ, Klevering BJ, Leroy BP, et al. The spectrum of ocular phenotypes caused by mutations in the BEST1 gene. Prog Retin Eye Res. 2009;28:187-205.

Hamel CP. Cone rod dystrophies. Orphanet J Rare Dis. 2007; 
8. Thiadens AA, Phan TM, Zekveld-Vroon RC, et al. Clinical course, genetic etiology, and visual outcome in cone and cone-rod dystrophy. Ophthalmology. 2012;119:819-26.

9. Molday RS, Kellner U, Weber BH. X-linked juvenile retinoschisis: clinical diagnosis, genetic analysis, and molecular mechanisms Prog Retin Eye Res. 2012; 31:195-212.

10. Sujirakul T, Lin MK, Duong J, et al. Multimodal imaging of centra retinal disease progression in a 2-year mean follow-up of retinitis pigmentosa. Am J Ophthalmol. 2015;160:786-98.

11. Pang $C E$, Suqin $Y$, Sherman J, Freund KB. New insights into Stargardt disease with multimodal imaging. Ophthalmic Surg Lasers Imaging Retina. 2015;46:257-61.

12. Battaglia Parodi $M$, lacono $P$. Romano $F$, Bandello F. Spectral domain optical coherence tomography features in different stages of Best vitelliform macular dystrophy. Retina. 2018;38:1041-6.

13. Pichi $F$, Morara $M$, Veronese $C$, et al. Multimodal imaging in hereditary retinal diseases. J Ophthalmol. 2013;2013:634351

14. Huang $Y$, Zhang $Q$, Thorell $M$, et al. Swept-source OCT angiography of the retinal vasculature using intensity differentiation-based optical microangiography algorithms. Ophthalmic Surg Lasers Imaging Retina. 2014;45:382-9.

15. Yannuzzi NA, Gregori NZ, Roisman L, et al. Fluorescein angiography versus optical coherence tomography angiography in macular telangiectasia type I treated with bevacizumab therapy. Ophthalmic Surg Lasers Imaging Retina. 2017:48:263-6.

16. Salz DA, de Carlo $T E$, Adhi M, et al. Select features of diabetic retinopathy on swept-source optical coherence tomographic angiography compared with fluorescein angiography and normal eyes. JAMA Ophthalmol. 2016;134:644-50.

17. Battaglia Parodi M, Pierro L, Gagliardi M, et al. Optica coherence tomography angiography in dystrophies Dev Ophthalmol. 2016;56:159-65.

18. Stanga PE, Papayannis A, Tsamis E, et al. Swept-source optical coherence tomography angiography of paediatric macular diseases. Dev Ophthalmol. 2016;56:166-73.

19. Battaglia Parodi M, Cicinelli MV, Rabiolo A, et al. Vessel density analysis in patients with retinitis pigmentosa by means of optical coherence tomography angiography. Br I Ophthalmol. 2017;101:428-32.

20. Koyanagi Y, Murakami Y, Funatsu J, et al. Optical coherence tomography angiography of the macular microvasculature tomography angiography of the macular microvascul changes in retinitis

21. Alnawaiseh M, Schubert F, Heiduschka P, Eter N. Optical coherence tomography angiography in patients with retinitis pigmentosa. Retina. 2017. DOI: 10.1097/IAE.0000000000001904
22. Battaglia Parodi M, Cicinelli MV, Rabiolo A, et al. Vascular abnormalities in patients with Stargardt disease assessed with optical coherence tomography angiography. Br J Ophthalmol. 2017;101:780-5.

23. Guduru A, Lupidi M, Gupta A, et al. Comparative analysis of autofluorescence and OCT angiography in Stargardt disease. Br J Ophthalmol. 2017 DOl: 10.1136/bjophthalmol-2017-311000.

24. Mastropasqua R, Toto L, Borrelli E, et al. Optical coherence tomography angiography findings in Stargardt disease. PLOS One. 2017:12:e0170343.

25. Battaglia Parodi M, Rabiolo A, Cicinelli MV, et al. Quantitative analysis of optical coherence tomography angiography in adultanalysis of optical coherence tomography angiography in adult-

26. Toto $L$, Borrelli E, Mastropasqua $R$, et al. Adult-onset foveomacular vitelliform dystrophy evaluated by means of optical coherence tomography angiography: a comparison with dry age-related macular degeneration and healthy eyes. Retina. 2017. DOI:10.1097/IAE 0000000000001615.

27. Treder M, Lauermann JL, Alnawaiseh M, et al. Quantitative changes in flow density in patients with adult-onset foveomacular vitelliform dystrophy: an OCT angiography study. Graefes Arch Clin Exp Ophthalmol. 2018;256:23-8.

28. Patel RC, Gao SS, Zhang M, et al. Optical coherence tomography angiography of choroidal neovascularization in four inherited retinal dystrophies. Retina. 2016;36:2339-47.

29. Battaglia Parodi M, Romano F Cicinelli MV, et al. Retinal vascular impairment in Best vitelliform macular dystrophy assessed by means of optical coherence tomography assessed by means of optical coherence tomograph

30. Abbouda A, Dubis AM, Webster AR, Moosajee M. Identifying characteristic features of the retinal and choroidal vasculature in choroideremia using optical coherence tomography angiography. Eye (Lond). 2018 Mar;32(3): 563-71.

31. Battaglia Parodi M, Arrigo A, MacLaren RE, et al. Vascular alterations revealed with optical coherence tomography angiography in patients with choroideremia. Retina. 2018 DOI: 10.1097//AE.0000000000002118 [Epub ahead of print].

32. Jain N, Jia Y, Gao SS, et al. Optical coherence tomography angiography in choroideremia: correlating choriocapillaris loss with overlying degeneration. JAMA Ophthalmol. 2016;134:697-702

33. Stringa F, Tsamis E, Papayannis A, et al. Segmented swept source optical coherence tomography angiography assessment of the perifoveal vasculature in patients with $\mathrm{X}$-linked juvenile retinoschisis: a serial case report. Int Med Case Rep J. 2017;10:329-35.

34. Weleber RG, Gregory-Evans K. Retinitis pigmentosa and allied disorders. In: Ryan SJ, Schachat A, Wilkinson C et al. Retina 4th ed., Philadelphia, PA: Elsevier, 2006;394-485.

35. Fishman GA, Stone EM, Grover S, et al. Variation of clinical expression in patients with Stargardt dystrophy and sequence variations in the ABCR gene. Arch Ophthalmol. 1999;117:504-10.

36. Walia S, Fishman GA. Natural history of phenotypic changes in Stargardt macular dystrophy. Ophthalmic Genet. 2009:30:63-8.

37. Gass JD. A clinicopathologic study of a peculiar foveomacular dystrophy. Trans Am Ophthalmol Soc. 1974;72:139-56.

38. Chowers I, Tiosano L, Audo I, et al. Adult-onset foveomacular vitelliform dystrophy: a fresh perspective. Progr Retin Eye Res. 2015;47:64-85.

39. Gass JDM. Best's disease. In: Gass J. Stereoscopic Atlas of Macular Diseases. Diagnosis and Treatment. 4th ed. Vol 1. St Louis, MO: Mosby; 1997:304-11.

40. Sorsby A, Franceschetti A, Joseph R, Davey JB. Choroideremia: clinical and genetic aspects. Br J Ophthalmol. 1952;36:547-81.

41. George ND, Yates JR, Moore AT. X linked retinoschisis. Br J Ophthalmol. 1995;79:697-702.

42. Apushkin MA, Fishman GA. Use of dorzolamide for patients with X-linked retinoschisis. Retina. 2006;26:741-5.

43. Lima LH, Cella W, Greenstein VC, et al. Structural assessment of hyperautofluorescent ring in patients with retinitis pigmentosa. Retina. 2009;29:1025-31

44. Eisenberger T, Neuhaus C, Khan AO, et al. Increasing the yield in targeted next-generation sequencing by implicating CNV analysis, non-coding exons and the overall variant load: the analysis, non-coding exons and the overall variant load: the

45. Parodi MB, lacono P, Del Turco C, Bandello F. Near-infrared fundus autofluorescence in subclinical best vitelliform macular dystrophy. Am J Ophthalmol. 2014;158:1247-52.

46. Ramsden CM, Powner MB, Carr AJ, et al. Stem cells in retinal regeneration: past, present and future. Development. 2013;140:2576-85.

47. Garg SJ, Federman J. Optogenetics, visual prosthesis and electrostimulation for retinal dystrophies. Curr Opin Ophthalmol. 2013;24:407-14.

48. Ghazi NG, Abboud EB, Nowilaty SR, et al. Treatment of retinitis pigmentosa due to MERTK mutations by ocular subretinal injection of adeno-associated virus gene vector results of a phase I trial. Hum Gene. 2016;135:327-43.

49. MacLaren RE, Groppe M, Barnard AR, et al. Retinal gene therapy in patients with choroideremia: initial findings from a phase 1/2 clinical trial. Lancet. 2014:383:1129-37.

50. Chen FK, Viljoen RD, Bukowska DM. Classification of image artefacts in optical coherence tomography angiography of the choroid in macular diseases. Clin Exp Ophthalmo 2016;44:388-99 